\title{
Factores asociados a morbilidad precoz en cirugía colorrectal laparoscópica*
}

\author{
Drs. JOSÉ GELLONA V. ${ }^{1}$, RODRIGO MIGUIELES C. ${ }^{1}$, DANIELLA ESPÍNOLA M. ${ }^{1}$, \\ GONZALO URREJOLA S. ${ }^{1}$, MARÍA E. MOLINA P. ${ }^{1}$, GONZALO INOSTROZA L. ${ }^{1}$, \\ JOSÉ M. ZÚÑIGA A. ${ }^{1}$, TOMÁS LARACH K. ${ }^{1}$, FELIPE BELLOLIO R. ${ }^{1}$, ÁLVARO ZÚÑIGA D. ${ }^{1}$
}

1 Unidad de Coloproctología, Departamento de Cirugía Digestiva, Facultad de Medicina, Pontificia Universidad Católica de Chile.

Santiago, Chile.

Abstract

Factors associated with early morbidity in laparoscopic colorectal surgery

Introduction: Different factors have been associated with increased risk of complications in laparoscopic colorectal surgery. The aim of this study is to identify these factors in our series. Method: Retrospective cohort. All patients undergoing laparoscopic colorectal surgery between January 2000 and June 2012 were included. Patients who had postoperative complications until 30 days postoperatively were identified and analyzed by univariate and multivariate logistic regression. A p value less than 0.2 was used was used as a criteria for entry into the multivariate model. Results: The series consists of 848 patients with a median age of $58 \pm 22$ years. Main surgical indications were: neoplasia (42.3\%), diverticular disease (27.8\%) and inflammatory bowel disease (8.8\%). Most frecuently-performed procedures were: sigmoidectomy (39.5\%), anterior resection of the rectum (13.4\%), right hemicolectomy (13\%) and total colectomy $(8.7 \%)$. On univariate analysis, factors associated with complications were age over 75 years (OR 1.82, 95\% CI 1.02 to 3.25) and red blood cell transfusion (OR 8.47, 95\% CI 3.69 to 19.43). On multivariate analysis, red blood cell transfusion (OR 7.9 95\% CI 1.78 to 35.88) and ASA III or IV (OR 3.26 95\% CI 1.01 to 17.23) were independent factors associated with postoperative complications. Conclusion: Intraoperative red blood cell transfusion and ASA score III or IV are independent risk factors associated with complications in laparoscopic colorectal surgery.

Key words: Colorectal, laparoscopic, morbidity, risk factors.

\section{Resumen}

Introducción: Se han descrito factores que se asocian a mayor riesgo de complicaciones en cirugía laparoscópica colorrectal. El objetivo de este trabajo es identificar estos factores en nuestra serie. Método: Cohorte histórica. Se incluyeron todos los pacientes sometidos a cirugía colorrectal laparoscópica entre enero de 2000 y junio de 2012. Se identificaron los pacientes que tuvieron complicaciones post operatorias hasta 30 días después de la operación. Se analizaron mediante regresión logística uni y multivariada. Se utilizó como criterio de entrada al modelo multivariado los $p<0,2$ y como criterio de significancia un $p=0,05$. Resulta-

*Recibido el 19 de marzo de 2013 y aceptado para publicación el 13 de abril de 2013.

Los autores no tienen conflictos de interés que comunicar.

Correspondencia: Dr. José Gellona V.

Marcoleta 350 Santiago. Chile

jgellona@yahoo.com 
dos: La serie consta de 848 pacientes, con una mediana de edad de $58 \pm 22$ años. Las principales indicaciones operatorias fueron: neoplasia (42,3\%), enfermedad diverticular $(27,8 \%)$ y enfermedad inflamatoria intestinal $(8,8 \%)$. Las operaciones realizadas con mayor frecuencia fueron: sigmoidectomía $(39,5 \%)$, resección anterior de recto $(13,4 \%)$, hemicolectomía derecha $(13 \%)$ y colectomía total $(8,7 \%)$. En el análisis univariado, los factores asociados a complicación fueron: la edad sobre 75 años (OR de 1,82; IC 95\% 1,02-3,25) y la transfusión de glóbulos rojos (OR 8,47; IC 95\% 3,69-19,43). En el análisis multivariado, la transfusión de glóbulos rojos (OR 7,9 95\% IC 1,78-35,88) y el ASA III o IV (OR 3,26 95\% IC 1,01-17,23) fueron factores de riesgo independientes de complicaciones en el postoperatorio. Conclusión: La necesidad de transfusión y el ASA III o IV son factores de riesgo independientes asociados a complicaciones en cirugía colorrectal laparoscópica.

Palabras clave: Colorrectal, laparoscópica, morbilidad, factores de riesgo.

\section{Introducción}

El abordaje laparoscópico en cirugía colorrectal está establecido en la mayoría de los centros de referencia como el abordaje de elección. Numerosas publicaciones han demostrado las ventajas del abordaje laparoscópico en cuanto a menor necesidad de analgésicos, menor tiempo de estadía hospitalaria, menor tiempo de reinicio de actividades regulares y menor infección de sitio quirúrgico ${ }^{1-5}$. Sin embargo, el abordaje mínimamente invasivo no está exento de complicaciones, su morbilidad descrita va entre un 6 $y$ un $30 \%$ y la mortalidad entre 0 y $4,5 \%{ }^{6}$.

Lograr encontrar factores predictivos o puntajes que permitan identificar a los pacientes de alto riesgo de complicación es una idea que se ha implementado anteriormente. Dentro de los índices validados se encuentran el puntaje CR- POSSUM ${ }^{7}$ y el índice de riesgo preoperatorio en cirugía colorrectal de Cleveland ${ }^{8}$. Sin embargo, estos puntajes no se han logrado validar y son difíciles de aplicar en la práctica diaria, además sólo predicen mortalidad postoperatoria. Por esta razón, varios factores se han estudiado e intentado asociar a complicación postoperatoria en cirugía colorrectal, entre ellos se encuentra la experiencia del cirujano, edad, género masculino, elevado puntaje de ASA preoperatorio, cirugía rectal, neoplasia y cirugía de urgencia entre otros ${ }^{6,9}$. Todos estos factores han sido asociados a complicación, sin embargo, la asociación varía mucho en las distintas series publicadas, lo que explica que modelos predictivos de un centro no puedan ser replicados en otro centro posteriormente ${ }^{10}$.

Por lo descrito anteriormente, dada la variabilidad de los resultados dependiendo de la serie estudiada, además de la necesidad de conocer en nuestro medio los factores asociados a morbilidad, este trabajo tiene por objetivo identificar factores asociados a complicación precoz postoperatoria en nuestra serie de cirugía laparoscópica colorrectal.

\section{Método}

Estudio de cohorte no concurrente. Los datos se obtuvieron de la base de datos de cirugía colorrectal laparoscópica llevada en forma prospectiva, la cual forma parte del protocolo de cirugía laparoscópica colorrectal iniciado en el año 2000. Se incluyeron todos los pacientes sometidos a procedimientos colorrectales laparoscópicos entre enero de 2000 y junio de 2012. Se excluyeron pacientes sometidos a re intervención por alguna complicación. Se identificaron los pacientes que presentaron complicaciones dentro de los 30 días postoperatorios y se analizaron de forma uni y multivariada los factores asociados a complicación.

Para la descripción de la morbilidad se utilizó la clasificación de Clavien y Dindo ${ }^{11}$, la que considera la magnitud de la complicación, el tratamiento requerido y su repercusión en el paciente, considerándose morbilidad menor a las complicaciones correspondientes al grado I o II y morbilidad mayor al grado III, IV o V de dicha clasificación

Para el análisis estadístico se utilizó estadística descriptiva, los datos numéricos están expresados en mediana (mínimo-máximo) dada su distribución; los datos categóricos se expresan en porcentajes. Para el análisis univariado se utilizó regresión logística binaria, obteniendo así los Odds Ratio (OR) para cada factor. Se realizó posteriormente el análisis mutivariado utilizando como criterio de entrada si el factor estudiado en el análisis univariado tuvo un $\mathrm{p}$ menor a 0,2 . Se comprobó la bondad de ajuste del modelo mediante la prueba de Hosmer y Lemeshow y se obtuvo el área bajo la curva (curva ROC). Se consideró valor significativo a un p menor a 0,05 en todos los análisis.

\section{Resultados}

Un total de 848 pacientes fueron sometidos a procedimientos laparoscópicos colorrectales en el período mencionado. De estos, $404(47,6 \%)$ eran de sexo masculino. La mediana de edad de la serie fue de 58 (18-94) años, con un índice de masa corporal (IMC) de 25,8 $(16,9-35,3) \mathrm{kg} / \mathrm{m}^{2}$. El 35,3\% de los pacientes tenía el antecedente de cirugía abdominal. Con respecto a la distribución del puntaje de ASA 
preoperatorio, la gran mayoría de los pacientes eran ASA I o II (Tabla 1).

Tal como se observa en la Tabla 2, la neoplasia, la enfermedad diverticular y la enfermedad inflamatoria intestinal dieron cuenta de $84 \%$ de las indicaciones operatorias en esta serie. La sigmoidectomía, resección anterior, hemicolectomía derecha y la colectomía total o subtotal fueron las 4 cirugías realizadas con mayor frecuencia, acumulando en conjunto el $74,6 \%$ de todos los procedimientos realizados; el detalle de las operaciones realizadas se muestra en la Tabla 3.

El tiempo quirúrgico fue de 180 (30-500) min. La estadía postoperatoria fue de 5 (259) días. El 87,4\% de las cirugías implicó la resección de algún segmento y en el 83,7\% del total de procedimientos se realizó una anastomosis. El 7,2\% de los pacientes fue convertido a cirugía tradicional. Un total de 100 pacientes presentaron alguna complicación, lo que corresponde al $11,8 \%$ de la serie. $\mathrm{Si}$ desglosamos por morbilidad menor y mayor vemos que el $8,1 \%$ del total de pacientes presentaron complicaciones menores y el 3,7\% mayores. Un paciente de la serie falleció, lo que corresponde al $0,1 \%$ del total. Un total de 22 pacientes debieron ser reoperados, lo que corresponde al 2,6\% de la serie estudiada. El detalle de las complicaciones mayores está expuesto en la Tabla 4.

En el análisis univariado, la necesidad de transfusión de glóbulos rojos en el intraoperatorio y la edad sobre 75 años, fueron los factores que resultaron significativos con un OR de $8,36(3,65-19,18)$ y $1,79(1,01-3,20)$ respectivamente. Los factores que no resultaron significativos pero que se consideraron para el análisis multivariado fueron el puntaje ASA III o IV, el IMC, el tiempo operatorio y la cirugía de urgencia (Tabla 5).

En el análisis multivariado, la transfusión de glóbulos rojos en el intraoperatorio mantuvo su significancia estadística, con un OR de 7,61 (1,67-34,57). El puntaje ASA III o IV, se mostró también un factor asociado a morbilidad con un OR de 3,83 (1,01-16,40). La edad perdió su asociación al ajustarla por los otros factores incorporados al modelo. La cirugía de urgencia, IMC y tiempo operatorio se mantuvieron como factores no significativos (Tabla 5). El área bajo la curva (curva ROC) de este modelo mostró un valor de 0,711 y la prueba de bondad de ajuste de Hosmer y Lemeshow fue de 0,341 , lo que indica que el modelo se ajusta correctamente a los valores observados.
Tabla 1. Características generales de la serie estudiada

\begin{tabular}{|llc|}
\hline Variable & & Resultado \\
Pacientes & $\mathrm{n}$ & 848 \\
Género masculino & $\mathrm{n}(\%)$ & $404(47,7)$ \\
Edad & & $58(18-94)$ \\
IMC & & $25,8(16,9-42,2)$ \\
Cirugía abdominal previa & $\mathrm{n}(\%)$ & $299(35,3)$ \\
Cirugía resectiva & $\mathrm{n}(\%)$ & $741(87,4)$ \\
Cirugía urgencia & $\mathrm{n}(\%)$ & $58(6,8)$ \\
Cirugía con anastomosis & & $710(83,7)$ \\
ASA preoperatorio & $(\%)$ & \\
$\quad$ I & & 39 \\
II & & 50,1 \\
II & & 6,4 \\
Sin información & & 4,5 \\
\hline
\end{tabular}

Tabla 2. Indicaciones operatorias de la serie

\begin{tabular}{|lrrc|}
\hline Indicación & n & \% & Acumulado \\
Neoplasia & 359 & 42,3 & 42,3 \\
Diverticular & 236 & 27,8 & 70,1 \\
Inflamatorio & 75 & 8,8 & 78,9 \\
Pólipos & 41 & 4,8 & 83,7 \\
Prolapso & 37 & 4,4 & 88,1 \\
Ostomía & 35 & 4,2 & 92,3 \\
Inercia/megacolon & 35 & 4,2 & 96,5 \\
Íleo mecánico/vólvulo & 18 & 2,1 & 98,6 \\
Otras & 12 & 1,4 & 100 \\
Total & 848 & 100 & 100 \\
\hline
\end{tabular}

Tabla 3. Operaciones realizadas

\begin{tabular}{|lrrc|}
\hline Operación & n & \% & Acumulado \\
Sigmoidectomía & 335 & 39,5 & 39,5 \\
Resección anterior & 114 & 13,4 & 52,9 \\
Hemicolectomía derecha & 110 & 13,0 & 65,9 \\
Colectomía total/subtotal & 74 & 8,7 & 74,6 \\
Reconstitución del tránsito & 46 & 5,4 & 80 \\
Hemicolectomía izquierda & 42 & 5,0 & 85 \\
Proctocolectomía & 29 & 3,4 & 88,4 \\
Pexias & 23 & 2,7 & 91,1 \\
Resección abdominoperineal & 21 & 2,5 & 93,6 \\
Ostomía & 16 & 1,9 & 95,5 \\
Otras & 36 & 4,5 & 100 \\
Total & 848 & 100 & 100 \\
\hline
\end{tabular}


Tabla 4. Detalle de las complicaciones mayores de la serie

\begin{tabular}{|lcc|}
\hline Complicación & $\mathbf{n}$ & $\mathbf{\%}$ \\
Colección & 8 & 0,94 \\
Filtración de anastomosis & 7 & $0,98^{*}$ \\
Hemoperitoneo & 5 & 0,58 \\
Íleo mecánico & 4 & 0,47 \\
Sepsis pélvica & 3 & 0,35 \\
Evisceración & 2 & 0,23 \\
Trombosis portal & 1 & 0,12 \\
Hematoma pared & 1 & 0,12 \\
\hline
\end{tabular}

*Este porcentaje corresponde al $0,98 \%$ de los pacientes con anastomosis de la serie

\section{Discusión}

La factibilidad y seguridad de la cirugía laparoscópica colorrectal se ha comunicado múltiples veces en la literatura quirúrgica actual. El resultado operatorio en términos de complicaciones puede ser más exitoso si conocemos factores que podrían afectar la evolución del paciente. Por esto se hace necesario poseer herramientas objetivas que permitan identificar a los pacientes con mayor riesgo de complicación.

La morbilidad y mortalidad de esta serie se encuentran en los límites inferiores de lo comunicado en la literatura ${ }^{6}$. Esto es el reflejo de un centro terciario de alta complejidad, con un relativo alto volumen

Tabla 5: Análisis univariado y multivariado de los factores estudiados

\begin{tabular}{|c|c|c|c|c|}
\hline \multirow[t]{2}{*}{ Variable $(n=848)$} & \multicolumn{2}{|c|}{ Univariado Odds Ratio } & \multicolumn{2}{|c|}{ Multivariado Odds Ratio } \\
\hline & (IC 95\%) & $\mathbf{p}$ & (IC 95\%) & $\mathbf{p}$ \\
\hline Transfusión GR intraoperatoria & $\begin{array}{c}8,36 \\
(3,65-19,18)\end{array}$ & 0,001 & $\begin{array}{c}7,61 \\
(1,67-34,57)\end{array}$ & 0,009 \\
\hline Edad (> 75 años) & $\begin{array}{c}1,79 \\
(1,01-3,20)\end{array}$ & 0,048 & $\begin{array}{c}1,31 \\
(0,45-3,85)\end{array}$ & 0,617 \\
\hline ASA III-IV & $\begin{array}{c}1,91 \\
(0,95-3,85)\end{array}$ & 0,068 & $\begin{array}{c}3,83 \\
(1,01-16,40)\end{array}$ & 0,049 \\
\hline IMC & $\begin{array}{c}0,94 \\
(0,86-1,02)\end{array}$ & 0,155 & $\begin{array}{c}0,96 \\
(0,87-1,05)\end{array}$ & 0,397 \\
\hline Tiempo operatorio & $\begin{array}{c}1,00 \\
(0,99-1,00)\end{array}$ & 0,174 & $\begin{array}{c}1,00 \\
(0,99-1,00)\end{array}$ & 0,713 \\
\hline Cirugía de urgencia & $\begin{array}{c}1,68 \\
(0,79-3,31)\end{array}$ & 0,186 & $\begin{array}{c}3,32 \\
(0,83-13,13)\end{array}$ & 0,087 \\
\hline Cirugía de recto & $\begin{array}{c}1,32 \\
(0,84-2,05)\end{array}$ & 0,232 & & \\
\hline Obesidad (IMC > 30) & $\begin{array}{c}0,62 \\
(0,20-1,86)\end{array}$ & 0,387 & & \\
\hline Neoplasia & $\begin{array}{c}1,17 \\
(0,77-1,79)\end{array}$ & 0,451 & & \\
\hline Cirugía abdominal previa & $\begin{array}{c}0,82 \\
(0,54-1,27)\end{array}$ & 0,383 & & \\
\hline Cirugía con anastomosis & $\begin{array}{c}0,76 \\
(0,43-1,23)\end{array}$ & 0,253 & & \\
\hline Conversión & $\begin{array}{c}1,52 \\
(0,74-3,09)\end{array}$ & 0,251 & & \\
\hline Cirugía resectiva & $\begin{array}{c}0,94 \\
(0,49-1,73)\end{array}$ & 0,841 & & \\
\hline Género (masculino) & $\begin{array}{c}0,97 \\
(0,64-1,48)\end{array}$ & 0,902 & & \\
\hline
\end{tabular}


de cirugía colorrectal laparoscópica; además los procedimientos fueron realizados por cirujanos colorrectales expertos o residentes de la subespecialidad directamente supervisados.

En esta serie de 848 pacientes, se estudiaron 14 factores perioperatorios, de los cuales 2 , la necesidad de transfusión de glóbulos rojos y el ASA III-IV son factores de riesgo independientes para complicación dentro de los primeros 30 días.

Los hallazgos mencionados anteriormente concuerdan parcialmente con otras series ya publicadas $^{6,9}$. La necesidad de transfusión de glóbulos rojos intraoperatoria fue el factor con mayor fuerza de asociación. Probablemente la presencia de este factor indique indirectamente una cirugía de mayor complejidad técnica o sea el reflejo de algún incidente intraoperatorio. Este factor se ha estudiado principalmente en cáncer colorrectal y sobrevida a largo plazo, donde los pacientes que reciben transfusión podrían tener peor sobrevida que aquellos que no la reciben ${ }^{12}$. En resultados a corto plazo, un reciente meta análisis mostró una asociación con mayores infecciones postoperatorias, necesidad de re operación, mayor tiempo de estadía postoperatoria y mortalidad por cualquier causa en pacientes que recibieron transfusión ${ }^{13}$. Por otro lado, el puntaje ASA es un factor conocido que ha sido asociado consistentemente con morbilidad y mortalidad perioperatoria en cirugía colorrectal tanto abierta como laparoscópica ${ }^{6,9}$, sin embargo, en una serie más reciente este factor no se asoció a complicación ${ }^{14}$.

La obesidad es otro factor comúnmente asociado a complicación en cirugía colorrectal laparoscópica. Zhou et al, en un reciente meta análisis ${ }^{15}$, encontraron un $34,8 \%$ de complicaciones en pacientes obesos comparado con un $23,4 \%$ en no obesos, especialmente complicaciones como íleo, infección de sitio quirúrgico y eventos pulmonares. Con respecto a filtración, abscesos, hemorragias y mortalidad no encontraron diferencias. En nuestra serie, se estudió el IMC como variable cuantitativa y además como variable categórica. No se encontró asociación con morbilidad en ninguna de las 2 formas. Probablemente la diferencia está dada en pacientes con obesidad severa o mórbida, tipo de pacientes que no hay en esta serie; como se mostró anteriormente, el valor máximo de IMC es de $35,3 \mathrm{~kg} / \mathrm{m}^{2}$. Esto se explica probablemente por el alto porcentaje de pacientes con cáncer y enfermedad inflamatoria intestinal presentes en esta serie.

La cirugía con anastomosis, la neoplasia y la cirugía resectiva no fueron asociadas a morbilidad precoz en este estudio, un hallazgo quizá inesperado sabiendo que son factores que deberían agregar mayor riesgo a la cirugía, tanto por el compromiso sistémico inmunitario del paciente con cáncer, el riesgo de lesiones asociadas a la resección y por la confección de anastomosis.

Las variables incluidas en el modelo se eligieron mediante un criterio estadístico, por lo que algunas variables que serían relevantes en la asociación con complicaciones no se incluyeron al análisis multivariado, por ejemplo, la cirugía resectiva y la cirugía con anastomosis. Al probar estas variables en el modelo multivariado se mantienen sin significancia y por otro lado, la bondad de ajuste y el área bajo la curva del modelo disminuyeron (datos no mostrados).

En conclusión, este estudio muestra que la necesidad de transfusión intraoperatoria y el ASA III o IV son factores de riesgo independientes para tener una complicación temprana en el postoperatorio. La presencia de estos factores en un paciente deberá ser un llamado a la preocupación y requerirán la mayor atención en el período postoperatorio.

\section{Referencias}

1.- Lezoche E, Feliciotti F, Guerrieri M, Paganini AM, De Sanctis A, Campagnacci R, et al. Laparoscopic versus open hemicolectomy. Minerva Chir. 2003;58:491-502.

2.- Scala A, Huang A, Dowson HM, Rockall TA. Laparoscopic colorectal surgery - results from 200 patients. Colorectal Dis. 2007;9:701-5.

3.- Feliciotti F, Guerrieri M, Paganini AM, De Sanctis A, Campagnacci R, Perretta S, et al. Long-term results of laparoscopic versus open resections for rectal cancer for 124 unselected patients. Surg Endosc. 2003;17:1530-5.

4.- Kang CY, Chaudhry OO, Halabi WJ, Nguyen V, Carmichael JC, Stamos MJ, et al. Outcomes of laparoscopic colorectal surgery: data from the Nationwide Inpatient Sample 2009. Am J Surg. 2012;204:952-7.

5.- Schwenk W, Haase O, Neudecker J, Muller JM. Short term benefits for laparoscopic colorectal resection. Cochrane Database Syst Rev. 2005:CD003145.

6.- Kirchhoff P, Dincler S, Buchmann P. A multivariate analysis of potential risk factors for intra- and postoperative complications in 1316 elective laparoscopic colorectal procedures. Ann Surg. 2008;248:259-65.

7.- Tekkis PP, Prytherch DR, Kocher HM, Senapati A, Poloniecki JD, Stamatakis JD, et al. Development of a dedicated risk-adjustment scoring system for colorectal surgery (colorectal POSSUM). Br J Surg. 2004;91:1174-82.

8.- Fazio VW, Tekkis PP, Remzi F, Lavery IC. Assessment of operative risk in colorectal cancer surgery: the Cleveland Clinic Foundation colorectal cancer model. Dis Colon Rectum 2004;47:2015-24.

9.- Ragg JL, Watters DA, Guest GD. Preoperative risk stratification for mortality and major morbidity in major colorectal surgery. Dis Colon Rectum 2009;52:1296303. 
10.- Dogrul AB, Kilic YA, Celebi AE, Celik S, Dumlu GE, Cetinkaya E, et al. External validation of Cleveland Clinic Foundation colorectal cancer model in a University Clinic in terms of predicting operative mortality. Tech Coloproctol. 2010;14:9-12.

11.- Dindo D, Demartines N, Clavien PA. Classification of surgical complications: a new proposal with evaluation in a cohort of 6336 patients and results of a survey. Ann Surg. 2004;240:205-13.

12.- Harlaar JJ, Gosselink MP, Hop WC, Lange JF, Busch $\mathrm{OR}$, Jeekel H. Blood transfusions and prognosis in colorectal cancer: long-term results of a randomized controlled trial. Ann Surg. 2012;256:681-6; discussion
686-7.

13.- Acheson AG, Brookes MJ, Spahn DR. Effects of allogenic red blood cell transfusions on clinical outcomes in patients undergoing colorectal cancer surgery: a systematic review and meta-analysis. Ann Surg. 2012;256:235-44.

14.- Manilich E, Vogel JD, Kiran RP, Church JM, SeyidovaKhoshknabi D, Remzi FH. Key factors associated with postoperative complications in patients undergoing colorectal surgery. Dis Colon Rectum. 2013;56:64-71.

15.- Zhou Y, Wu L, Li X, Wu X, Li B. Outcome of laparoscopic colorectal surgery in obese and nonobese patients: a meta-analysis. Surg Endosc. 2012;26:783-9. 